•编者按・

\title{
中国野生动物红外相机监测网络建设进展与展望
}

\author{
李 戒 $1,2^{*}$ \\ 1 (北京大学生命科学学院, 北京 100871) \\ 2 (北京大学生态研究中心, 北京 100871)
}

\section{Development progress and outlook of the wildlife camera-trapping networks in China}

Sheng $\mathrm{Li}^{1,2^{*}}$

1 School of Life Sciences, Peking University, Beijing 100871

2 Institute of Ecology, Peking University, Beijing 100871

自20世纪90年代以来，红外相机技术在野生动 物调查、研究与监测中逐渐得到了广泛的应用(李晟 等, 2014)。我国的红外相机应用与国际同步, 在过 去10年间快速发展，目前已成为地栖大中型兽类与 鸟类多样性监测中应用最为广泛的技术之一(李晟 等, 2014; 朱淑怡等, 2017), 为我国野生动物的生物 多样性编目、监测提供了大量的实地观测数据(肖治 术等, 2014; 肖治术, 2019)。截至2019年底, 我国已 建立十多个区域性或全国性红外相机监测网络或 监测平台。本期汇总了其中 10 个监测网络的文章, 详细描述了各网络的建立历史、覆盖区域、监测目 标等基本信息, 统计了各网络截至2019年的调查工 作量、数据量以及记录到的兽类、鸟类物种名录, 并 总结了各自的主要监测成果, 对后续的重点工作方 向进行了梳理。

通过总结, 我们可以看到, 在过去20年间, 中 国的野生动物红外相机监测网络建设具有以下几 方面的特点:

(1)建设与参与单位广泛且多样, 网络管理、运 行机制各具特点。本期涉及的 10 个监测网络的牵头 与组织单位包括科研院所、高等院校、NGO、国家 公园等, 网络内参与单位还包括自然保护区、林场、 社区保护地、国外机构与组织等。监测样区大多依 托现有自然保护区、国家公园等保护地来设置, 并 与保护地自身的监测体系相结合。红外相机监测的
野外工作由科研工作者、高校学生、保护地职工、 当地社区居民、志愿者等多方力量共同承担，不同 网络内的管理机制、运行方式、数据汇总模式不尽 相同，各自摸索出了适合自身的运行机制。

(2)总体取得长足发展，工作量与数据量可观。 本期中 10 个监测网络合计在 238 个监测样区设置了 约1.7万个红外相机调查位点(图1), 布设红外相机 约 2 万台，截至 2019 年已获得了超过 1,100 万份的红 外相机照片(含视频。不包括未处理数据), 总工作量 超过 750 万相机日(附录1)。除这 10 个监测网络之外, 我国还建立有其他一些区域性或全国性红外相机 监测网络(例如，肖治术等，2014，2017), 同时还有 大量的保护地、机构组织与单位在开展局地的红外 相机调查与监测。据笔者粗略估计, 全国目前运行 的红外相机约在5万台以上。就国家尺度而言, 我国 的红外相机监测网络已成为全球最大的监测网络 之一 ; 以 eMammal (http://www.emammal.si.edu, McShea et al, 2016) 、 Wildlife Insights (http://www.wildlifeinsights.org, Ahumada et al, 2020) 等国际红外相机数据平台作为参考(Steenweg et al, 2017), 中国红外相机监测网络所积累的数据量与 数据集规模也已位于全球前列。

(3)监测网络之间开展了多种形式的合作，并 开展多方国际合作与交流。例如, 由生态环境部南 京环境科学研究所牵头建设的“中国哺乳动物多

* 通讯作者 Author for correspondence. E-mail: shengli@pku.edu.cn 


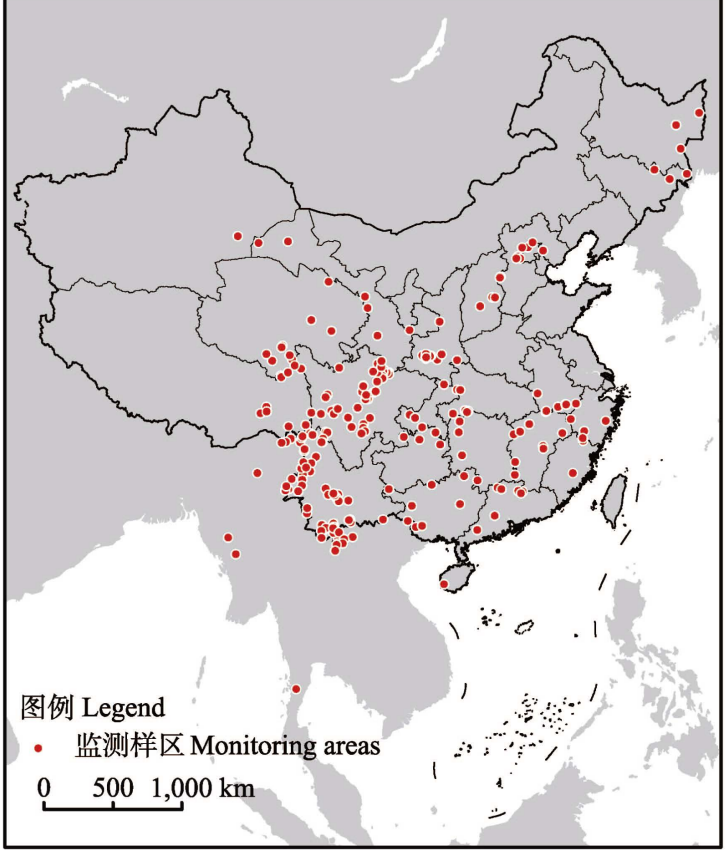

图1 中国10个红外相机监测网络的监测样区分布

Fig. 1 The monitoring areas of the 10 camera-trapping networks in China

样性观测网络”与其他多个监测网络有交集, 在部 分监测样区共同开展红外相机监测工作; 由北京大 学牵头建立的“西南山地红外相机监测网络”与 eMammal红外相机数据库(McShea et al, 2016)以合 作项目的形式在部分监测样区开展共同的红外相 机调查, 两个网络共享其中的部分数据; 由中国科 学院西双版纳热带植物园主持的“中国滇南-东南亚 跨境动物多样性监测平台”, 与东南亚的缅甸、老挝 等国的林业部门和保护区共同合作, 联合开展红外 相机监测。这些合作与交流为今后进一步推动红外 相机监测数据标准化、数据分享与平台合作摸索了 经验，奠定了基础。

(4)积累的监测数据极大地促进了我国兽类与 鸟类生物多样性编目和本底调查工作。截至2019年, 这10个监测网络在我国共记录到分属 11 目 32 科的 165 个兽类物种, 占我国野生哺乳动物物种总数 (692种, 蒋志刚等, 2017)的23.84\%; 记录到分属17 目61科的507个鸟类物种, 占我国野生鸟类物种总 数(1,445种, 郑光美, 2017)的35.09\%。监测中记录到 的缅甸斑羚 (Naemorhedus evansi)、白规猕猴 (Macaca leucogenys)、大斑灵猫(Viverra megaspila)、 小齿狸(Arctogalidia trivirgata)、灰腹角雉(Tragopan blythii)等物种(详见本期各篇监测网络文章的物种 名录)为我国近年来新记录种或罕见记录种，同时 也为许多省区和保护地增加了诸多兽类和鸟类物 种分布新记录。目前, 红外相机技术已成为我国地 栖大中型兽类与雉类等大型地栖鸟类野外调查与 监测的最有效工具之一。基于这些网络的监测工作, 我国的众多自然保护区、国家公园都针对各自区内 的兽类、鸟类开展了生物多样性编目工作，为完善 保护地的生物多样性本底做出了重要贡献。本期收 录了长江正源沱沱河流域以及广东鼎湖山、广东云 开山和甘肃安西极早荒漠等自然保护区的红外相 机研究报告, 均为此类案例。

(5)各监测网络近年来在物种分类、生态研究与 保护管理等多方面取得众多进展与成果(详见本期 各篇监测网络文章)。例如，由北京师范大学主持建 立的“东北虎豹生物多样性红外相机监测平台”首次 系统评估了中国东北虎(Panthera tigris altaica)与东 北豹( $P$. pardus orientalis)的种群数量, 为 2 个物种的 保护及东北虎豹国家公园的建立和管理提供了重 要的科学基础; 由中国科学院昆明动物研究所主持 的“西南纵向岭谷区红外相机监测网络”发现我国西 南干热河谷景观具有丰富且独特的野生动物多样 性, 并证实藏族神山为许多受威胁兽类提供了庇护; 由北京大学牵头建设的“西南山地红外相机监测网 络”则系统评估了大熊猫(Ailuropoda melanoleuca) 分布区内大型食肉动物的现状, 发现区内目前分布 有豹(Panthera pardus)、雪豹(P. uncia)、狼(Canis lupus)和然才(Cuon alpinus)等4种大型食肉动物, 但其 分布区范围自20世纪中期以来均出现明显下降，从 而建议当前正在建设的大熊猫国家公园应考虑将 恢复大型食肉动物种群作为长期管理目标之一，以 重建区域内生态系统的营养级复杂度, 增强其对外 部干扰的抵抗力。

今后, 中国的野生动物红外相机监测网络在进 一步的建设与发展中, 应着重关注以下方面:

(1)构建标准化的元数据结构, 建立网络平台 之间数据交流与分享的机制，促进数据交流。如果 要基于红外相机监测数据全面探明中国的生物多 样性现状与动态，或开展大尺度的生物多样性格局 研究, 各监测网络之间就需要深入开展数据分享与 交流。这样的合作将会极大地促进相关研究的深入 
开展, 推动多方合作, 共同取得更多、更重要的研 究成果。本期中, McShea等(2020)就这一问题进行 了深入探讨, 他们在回顾国内与国际已有经验的基 础上, 对数据标准、数据分享机制等提出了相应的 建议。

(2)开展深入的科学研究, 包括大尺度生物多 样性评估, 动物群落结构、种间关系与生态功能研 究等。在红外相机监测网络建设初期, 相关研究大 多集中在物种编目、动物群落多样性组成、单物种 栖息地分析等较浅层次。随着近年来监测网络的快 速发展, 这些网络所积累的大量数据, 可以为相关 科学研究和管理应用提供非常有价值的数据来源; 而大尺度的生物多样性评估、深入的动物群落和动 物生态学研究, 也可以依托已有的红外相机监测网 络来进行设计与实施。目前, 我国各红外相机监测 网络均已开始在多个方向开展更为深入的研究。可 以预期, 这些监测网络今后将为高质量研究成果的 持续产出提供有力支撑。

(3)推动新技术研发与应用, 包括前端数据自 动化采集与后端数据自动化处理、分析。随着红外 相机设备的不断完善与升级, 以及各方在红外相机 监测上投入的持续增长, 近年来各红外相机监测网 络内的数据量均迅速增加, 传统的数据采集、存储、 处理及分析方式已无法跟上数据累积的速度。目前, 研究者与工程师们正在把物联网、数据自动传输、 影像自动化识别、大数据存储与分析等先进理念、 技术与红外相机监测相结合 (Norouzzadeh et al, 2018), 同时也在开发更高效、更可靠的抽样方案与 数理模型(Kays et al, 2020), 以用于红外相机数据相 关的生态分析。未来, 这些多方面的技术进步与发 展, 以及跨领域的技术融合, 将进一步推动红外相 机监测的应用与发展。

(4)完善国家层面顶层设计, 合理布局监测样 区, 开发标准化监测规程, 建立中国红外相机监测 网络联盟。我国红外相机监测样区目前还存在空间 分布不均匀、缺乏系统的总体规划等问题。在国家 层面, 相关主管部门除了需要考虑完善监测样区科 学布局的顶层设计之外, 还需要考虑开发能够兼顾 不同生态区差异的标准化红外相机监测规程, 并组 织、协调现有各监测网络共同建立中国红外相机监 测网络联盟, 开发相应的大数据分享、交流平台。
(5)服务国家政策与工程，服务生态文明建设， 支持国家生物多样性保护、可持续发展进程与成效 评估。中国目前正在全面开展生态文明建设，众多 的相关政策、工程与项目，都需要可靠的、长期的 生物多样性监测数据与相应的评估指标体系的支 持。在以国家公园为主体的自然保护地体系建设、 中国参与的生物多样性相关国际条约的履约进程 评估等重要工作中, 红外相机监测网络所积累的大 数据具有巨大的潜力, 可用于计算动物种群、群落 等多尺度的生物多样性指标, 进而用于可靠地评估 我国区域性或全国性的生物多样性现状、动态与保 护成效, 为我国的生态文明建设、国际合作与谈判 等提供科学支撑与决策支持。

\section{参考文献}

Ahumada JA, Fegraus E, Birch T, Flores N, Kays R, O’Brien TG, Palmer J, Schuttler S, Zhao JY, Jetz W, Kinnaird M, Kulkarni S, Lyet A, Thau D, Duong M, Oliver R, Dancer A (2020) Wildlife Insights: A platform to maximize the potential of camera trap and other passive sensor wildlife data for the planet. Environmental Conservation, 47, 1-6.

Jiang ZG, Liu SY, Wu Y, Jiang XL, Zhou KY (2017) China's mammal diversity (2nd edition). Biodiversity Science, 25, 886-895. (in Chinese with English abstract) [蒋志刚, 刘少 英, 吴毅, 蒋学龙, 周开亚 (2017) 中国哺乳动物多样性 (第2版). 生物多样性, 25, 886-895.]

Kays R, Arbogast BS, Baker-Whatton M, Beirne C, Boone HM, Bowler M, Burneo SF, Cove MV, Ding P, Espinosa S, Goncalves ALS, Hansen CP, Jansen PA, Kolowski JM, Knowles TW, Lima MGM, Millspaugh J, McShea WJ, Pacifici K, Parsons AW, Pease BS, Rovero F, Santos F, Schuttler SG, Sheil D, Si X, Snider M, Spironello WR (2020) An empirical evaluation of camera trap study design: How many, how long and when? Methods in Ecology and Evolution, 11, 700-713.

Li S, Wang DJ, Xiao ZS, Li XH, Wang TM, Feng LM, Wang $Y$ (2014) Camera-trapping in wildlife research and conservation in China: Review and outlook. Biodiversity Science, 22, 685-695. (in Chinese with English abstract) [李 晟, 王大军, 肖治术, 李欣海, 王天明, 冯利民, 王云 (2014) 红外相机技术在我国野生动物研究与保护中的应 用与前景. 生物多样性, 22, 685-695.]

McShea WJ, Forrester T, Costello R, He Z, Kays R (2016) Volunteer-run cameras as distributed sensors for macrosystem mammal research. Landscape Ecology, 31, 55-66.

McShea WJ, Shen XL, Liu F, Wang TM, Xiao ZS, Li S (2020) China's wildlife camera-trap monitoring needs a unified standard. Biodiversity Science, 28, 1125-1131. (in Chinese 
and in English) [McShea WJ, 申小莉, 刘芳, 王天明, 肖 治术, 李晟 (2020) 中国的野生动物红外相机监测需要 统一的标准. 生物多样性, 28, 1125-1131.]

Norouzzadeh MS, Nguyen A, Kosmala M, Swanson A, Palmer MS, Packer C, Clune J (2018) Automatically identifying, counting, and describing wild animals in camera-trap images with deep learning. Proceedings of the National Academy of Sciences, USA, 115, 5716-5725.

Steenweg R, Hebblewhite M, Kays R, Ahumada J, Fisher JT, Burton C, Townsend SE, Carbone C, Rowcliffe JM, Whittington J, Brodie J, Royle JA, Switalski A, Clevenger AP, Heim N, Rich LN (2017) Scaling-up camera traps: Monitoring the planet's biodiversity with networks of remote sensors. Frontiers in Ecology and the Environment, $15,26-34$.

Xiao ZS, Li XH, Wang XZ, Zhou QH, Quan RC, Shen XL, Li S (2014) Developing camera-trapping protocols for wildlife monitoring in Chinese forests. Biodiversity Science, 22, 704-711. (in Chinese with English abstract) [肖治术, 李欣 海, 王学志, 周岐海, 权瑞昌, 申小莉, 李晟 (2014) 探 讨我国森林野生动物红外相机监测规范. 生物多样性, 22, 704-711.]

Xiao ZS, Li XY, Xiang ZF, Li M, Jiang XL, Zhang LB (2017) Overview of the Mammal Diversity Observation Network of
Sino BON. Biodiversity Science, 25, 237-245. (in Chinese with English abstract) [肖治术, 李学友, 向左甫, 李明, 蒋 学龙, 张礼标 (2017) 中国兽类多样性监测网的建设规 划与进展. 生物多样性, 25, 237-245.]

Xiao ZS (2019) Application of camera trapping to species inventory and assessment of wild animals across China's protected areas. Biodiversity Science, 27, 235-236. (in Chinese) [肖治术 (2019) 红外相机技术在我国自然保护 地野生动物清查与评估中的应用. 生物多样性, 27 , 235-236.]

Zheng GM (2017) A Checklist on the Classification and Distribution of the Birds of China, 3rd edn. Science Press, Beijing. (in Chinese) [郑光美 (2017) 中国鸟类分类与分 布名录(第三版). 科学出版社, 北京.]

Zhu SY, Duan F, Li S (2017) Promoting diversity inventory and monitoring of birds through the camera-trapping network in China: Status, challenges and future outlook. Biodiversity Science, 25, 1114-1122. (in Chinese with English abstract) [朱淑怡, 段菲, 李晟 (2017) 基于红外 相机网络促进我国鸟类多样性监测: 现状、问题与前景. 生物多样性, 25, 1114-1122.]

\section{附录 Supplementary Material}

附录 1 中国 10 个红外相机监测网络基本信息与数据量(截至 2019 年)

Appendix 1 Basic information and amount of data accumulated of the 10 camera-trapping networks in China (updated by 2019) http://www.biodiversity-science.net/fileup/PDF/2020425-1.pdf 
附录 1 中国 10 个红外相机监测网络基本信息与数据量 (截至 2019 年)

Appendix 1 Basic information and amount of data accumulated of the 10 camera-trapping networks in China (updated by 2019)

\begin{tabular}{|c|c|c|c|c|c|c|c|c|c|c|}
\hline 编号 ID & 网络名称 Networks* & $\begin{array}{c}\text { 牵头建设单位 } \\
\text { Leading organizations }\end{array}$ & $\begin{array}{c}\text { 建立年份 } \\
\text { Year } \\
\text { initiated }\end{array}$ & $\begin{array}{l}\text { 样区数 } \\
\text { Number of } \\
\text { monitoring } \\
\text { areas }\end{array}$ & $\begin{array}{c}\text { 相机位 } \\
\text { 点数 } \\
\text { Number } \\
\text { of } \\
\text { camera } \\
\text { stations }\end{array}$ & $\begin{array}{c}\text { 有效相机日 } \\
\text { (万天) } \\
\text { Number of } \\
\text { camera-days } \\
\left(\mathrm{x} 10^{4}\right)\end{array}$ & $\begin{array}{c}\text { 照片数(万份) } \\
\text { Number of } \\
\text { images }\left(\times 10^{4}\right)^{* *}\end{array}$ & $\begin{array}{l}\text { 兽类物种数 } \\
\text { Number of } \\
\text { mammal } \\
\text { species** }\end{array}$ & $\begin{array}{c}\text { 鸟类物种数 } \\
\text { Number of } \\
\text { bird } \\
\text { species** }\end{array}$ & $\begin{array}{l}\text { 参考文献 } \\
\text { Reference }\end{array}$ \\
\hline 1 & $\begin{array}{l}\text { 西南山地红外相机监测网 } \\
\text { 络 }\end{array}$ & 北京大学 & 2002 & 41 & 5738 & 120.7 & 302.6 & 63 & 182 & \multirow{10}{*}{$\begin{array}{c}\text { 李晟等, } \\
2020 \\
\text { 王天明 } \\
\text { 等, } 2020 \\
\text { 刘炎林 } \\
\text { 等, } 2020 \\
\text { 刘雪华 } \\
\text { 等, } 2020 \\
\text { 李佳等, } \\
2020 \\
\text { 李学友 } \\
\text { 等, } 2020 \\
\text { 贺如川 } \\
\text { 等, } 2020 \\
\text { 贾丁等, } \\
2020 \\
\text { 申小莉 } \\
\text { 等, } 2020 \\
\text { 万雅琼 } \\
\text { 等, } 2020\end{array}$} \\
\hline 2 & $\begin{array}{l}\text { 东北虎豹生物多样性红外 } \\
\text { 相机监测平台 }\end{array}$ & 北京师范大学 & 2006 & 6 & 910 & 173.6 & 78.5 & 28 & 32 & \\
\hline 3 & $\begin{array}{l}\text { 中国猫科动物红外相机监 } \\
\text { 测平台 }\end{array}$ & $\begin{array}{l}\text { 中国猫科动物保护联 } \\
\text { 盟 }\end{array}$ & 2007 & 28 & 939 & 28.3239 & 25 & 91 & 102 & \\
\hline 4 & $\begin{array}{l}\text { 秦岭中段野生动物多样性 } \\
\text { 红外相机监测数据库平台 }\end{array}$ & 清华大学 & 2009 & 4 & 267 & 15.216 & 85.526 & 27 & 63 & \\
\hline 5 & $\begin{array}{l}\text { 自然保护区标本资源共享 } \\
\text { 子平台红外相机数据库 }\end{array}$ & 中国林业科学研究院 & 2010 & 13 & 1667 & 27.25 & 16.34 & 80 & 200 & \\
\hline 6 & $\begin{array}{l}\text { 西南纵向岭谷区兽类及雉 } \\
\text { 类红外相机监测 }\end{array}$ & 中科院昆明动物所 & 2011 & 35 & 803 & 37.92 & 26.99 & 60 & 15 & \\
\hline 7 & $\begin{array}{l}\text { 中国滇南-东南亚跨境动 } \\
\text { 物多样性监测平台 }\end{array}$ & $\begin{array}{l}\text { 中科院西双版纳热带 } \\
\text { 植物园 }\end{array}$ & 2012 & 22 & 1493 & 9.7444 & 71.8995 & 48 & 80 & \\
\hline & $\begin{array}{l}\text { 三江源红外相机社区监测 } \\
\text { 平台 }\end{array}$ & $\begin{array}{l}\text { 北京大学, 北京山水 } \\
\text { 自然保护中心 }\end{array}$ & 2013 & 9 & 284 & 7.5 & 252.46 & 30 & 37 & \\
\hline 9 & $\begin{array}{l}\text { 钱江源国家公园红外相机 } \\
\text { 监测平台 }\end{array}$ & $\begin{array}{l}\text { 中科院植物研究所, } \\
\text { 钱江源国家公园 }\end{array}$ & 2014 & 6 & 1033 & 33.1834 & 71.8515 & 23 & 75 & \\
\hline 10 & $\begin{array}{l}\text { 中国哺乳动物多样性观测 } \\
\text { 网络平台 }\end{array}$ & $\begin{array}{l}\text { 生态环境部南京环科 } \\
\text { 所 }\end{array}$ & 2017 & 74 & 4440 & 300 & 200 & 132 & 407 & \\
\hline & 合计 Total & & & 238 & 17,574 & 753.4377 & 1131.167 & 165 & 507 & \\
\hline
\end{tabular}

*按各网络建立先后时间排序 Listed in the order according to the initiated year of each network.

**不包括未处理、未鉴定数据 Not include the raw data that have not been processed and identified. 


\section{参考文献}

He RC, Wang L, Quan RC (2020) Introduction to Transboundary Animal Diversity Monitoring Platform of Southern Yunnan, China and Southeast Asia. Biodiversity Science, 28, 1097-1103. (in Chinese with English abstract) [贺如川, 王林, 权锐昌 (2020) 中国滇南-东南亚跨境动物多样性监测平台概述. 生物多样性, 28, 1097-1103.]

Jia D, Li PY, Zhao X, Cheng C, Xiao LY, Lü Z (2020) Overview of Sanjiangyuan Community-based Cam-era- trapping Monitoring Platform. Biodiversity Science, 28, 1104-1109. (in Chinese with English abstract) [贾丁, 李沛芸, 赵翔, 程琛, 肖凌云, 吕植 (2020) 三江源红外相机社区监测平台概述. 生物多样性, 28, 1104-1109.]

Li J, Wang XL, Yang MW, Chen DX, Wang XJ, Luo P, Liu F, Xue YD, Li GL, Zhang YG, Zhang Y, Li DQ (2020) Construction progress of camera-trapping database from the Nature Reserves Biological Specimen Resources Sharing Sub-platform. Biodiversity Science, 28, 1081-1089. (in Chinese with English abstract) [李佳，王秀否，杨明伟，陈大祥，王晓菊，罗平，刘芳，薛亚 东, 李广良, 张于光, 张宇, 李迪强 (2020) 自然保护区生物标本资源共享子平台红外相机数据库建设进展. 生物多样性, 28, 1081-1089.]

Li S, McShea WJ, Wang DJ, Shen XL, Bu HL, Guan TO, Wang F, Gu XD, Zhang XF, Liao HH (2020) Construction progress of the Camera-trapping Net-work for the Mountains of Southwest China. Biodiversity Science, 28, 1049-1058. (in Chinese with English abstract) [李晟, William J. McShea，王大军，申小莉，卜红亮，官天培，王放，古晓东，张晓峰，廖激泓 (2020) 西 南山地红外相机监测网络建设进展. 生物多样性, 28, 1049-1058.]

Li XY, Hu WQ, Pu CZ, Li Q, Yu QP, Hu ZC, Bleisch WV, Jiang XL (2020) Camera-trapping monitoring platform for mammals and pheasants in the Longitudinal Range and Gorge Region of Southwest China: Protocol, progress and future outlook. Biodiversity Science, 28, 1090-1096. (in Chinese with English abstract) [李学友，胡文强，普昌哲，李权，于秋鹏，胡哲畅, William V. Bleisch, 蒋学龙 (2020) 西南纵向岭谷区兽类及雉类红外相机监测平台: 方案、进展与前景. 生物多样性, 28, 1090-1096.]

Liu XH, Zhang YK, Zhao XY, He XB, Cai Q, Zhu Y, He BS, Jiu Q (2020) Introduction to the wildlife camera-trapping data-base of the middle Qinling Mountains. Biodiversity Science, 28, 10751080. (in Chinese with English abstract) [刘雪华, 张语克, 赵翔宇, 何祥博, 蔡琼, 朱云, 何百锁, 酒强 (2020) 生物多样性, 28, 1075-1080.]

Liu YL, Song DZ, Liu BB, Xia F, Chen YL, Wang YQ, Huang QW (2020) Overview of the Camera-trapping Platform for Felid Species in China: Data integration by a conservation NGO. Biodiversity Science, 28, 1067-1074. (in Chinese with English abstract) [刘炎林，宋大昭，刘蓓蓓，夏凡，陈月龙，王一晴，黄巧雯 (2020) 中国猫科动物红外相机监测平台介绍: 民间 环保机构的数据整合. 生物多样性, 28, 1067-1074.]

Shen XL, Yu JP, Li S, Xiao HY, Chen XN, Chen SW, Liu MZ, Ma KP (2020) Progress overview of the camera-trapping monitoring platform for the Qianjiangyuan National Park, Zhejiang Province. Biodiversity Science, 28, 1110-1114. (in Chinese with English abstract) [申小莉, 余建平, 李晟, 肖慧芸, 陈小南, 陈声文, 刘鸣章, 马克平 (2020) 钱江源国家公园红外相机 监测平台进展概述. 生物多样性, 28, 1110-1114.]

Wan YQ, Li JQ, Yang XW, Li S, Xu HG (2020) Progress of the China mammal diversity observation network (China BON-Mammal) based on camera- trapping. Biodiversity Science, 28, 11151124. (in Chinese with English abstract) [万雅琼, 李佳琦, 杨兴文, 李晟, 徐海根 (2020) 基于红外相机的中国哺乳动物多样性观测网络建设进展. 生物多样性, 28, 1115-1124.]

Wang TM, Feng LM, Yang HT, Bao L, Wang HF, Ge JP (2020) An introduction to Long-term Tiger-Leopard Ob-servation Network based on camera traps in North-east China. Biodiversity Science, 28, 1059-1066. (in Chinese with English abstract) [王天明, 冯利民, 杨海涛, 鲍蕾, 王红芳, 葛剑平 (2020) 东北虎豹生物多样性红外相机监测平台概述. 生物多样性, 28, 1059-1066.] 